\title{
ESTUDIO COMPARATIVO ENTRE BIOAPATITA E HIDROXIAPATITA SINTÉTICA OBTENIDA POR PRECIPITACIÓN QUÍMICA Y SÍNTESIS MECANO- QUÍMICA
}

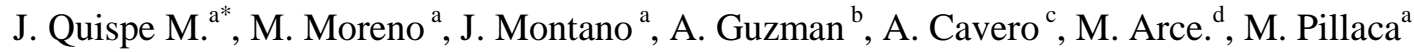 \\ ${ }^{a}$ Facultad de Ciencias Físicas, Universidad Nacional Mayor de San Marcos Ap. Postal 14-0149, Lima 14, Perú. \\ ${ }^{b}$ Facultad de Química e Ingeniería Química. Universidad Nacional Mayor de San Marcos. Perú. \\ "Unidad de Neurocirugía Pediátrica. Hospital "Edgardo Rebagliati Martins". \\ ${ }^{d}$ Facultad de Odontología. Universidad Santa Maria. Arequipa.
}

(Recibido Abril 01, 2009; Aceptado Setiembre 13, 2009)

\begin{abstract}
Resumen
Se presenta un estudio comparativo entre la componente inorgánica de una muestra de tejido óseo humano respecto de apatitas sintetizadas mediante precipitación química, síntesis mecanoquímica y una muestra de hidroxiapatita comercial. Las muestras fueron estudiadas mediante difracción de rayos X, espectroscopia por absorción atómica y espectroscopia infrarroja con transformada de Fourier. Los resultados obtenidos muestran características estructurales similares entre todas las muestras, identificándose que la muestra obtenida por síntesis mecanoquímica es un tipo de hidroxiapatita el cual presenta sustituciones de carbonato en su estructura cristalina similar a la componente inorgánica del tejido óseo.
\end{abstract}

PACS: $61.05 . \mathrm{cm} ;$ 87.64.Bx

Palabras claves: Bioapatita, hidroxiapatita, reacción mecano-química, difracción de rayos X.

\begin{abstract}
A comparative study between the inorganic component of a human bone tissue with respect of apatite synthesized by chemical precipitation, mechanochemical synthesis and a sample of commercial hidroxyapatite are shown. The samples were studied by X-ray diffraction, atomic absorption spectroscopy and Fourier transform infrared spectroscopy. The results show similar structural characteristics among all samples identifying that sample prepared by mechanochemical synthesis is a kind of hydroxyapatite which has substitutions of carbonate in its crystalline structure, similar to the inorganic component of bone tissue.
\end{abstract}

Keywords: Bioapatite, hydroxyapatite, mechano-chemical reaction, X-ray diffraction.

\section{Introducción}

Los huesos son tejidos duros mineralizados que permiten la movilidad del cuerpo y protegen los órganos internos blandos de todos los vertebrados. La principal componente inorgánica de estos tejidos duros (bioapatita) es una fase mineral de fosfato de calcio cuya estructura se parece a la estructura de la hidroxiapatita (Hap), $\mathrm{Ca}_{10}\left(\mathrm{PO}_{4}\right)_{6}(\mathrm{OH})_{2}$, conteniendo iones como impurezas en su estructura tales como $\mathrm{CO}_{3}{ }^{2-}, \mathrm{HPO}_{4}{ }^{2-}, \mathrm{SiO}_{4}{ }^{3-}, \mathrm{F}^{-}, \mathrm{Cl}^{-}, \mathrm{Mg}^{2+}, \mathrm{Na}^{+}, \mathrm{K}^{+}, \mathrm{y}$ algunos elementos trazas $\left(\mathrm{Sr}^{+2}, \mathrm{Zn}^{+2}\right)[1]$. En este sentido el estudio de la hidroxiapatita (Hap) ha despertado el interés de muchos grupos de investigación debido a que sus propiedades químicas y estructurales son similares a la componente inorgánica de los tejidos duros tanto de humanos como de animales [2]. Entre sus múltiples aplicaciones podemos mencionar su empleo como relleno y matriz ósea [3], regeneración de tejido óseo utilizando factores de crecimiento [4-6], así como intercambiadores iónicos en la purificación de aguas [7]. En la síntesis de Hap se han buscado diferentes alternativas como materia prima: desde huesos de animales [8] hasta fuentes naturales de carbonatos de calcio [9]. De manera similar se han empleado diferentes métodos de síntesis de Hap, tales como precipitación de soluciones acuosas [10], método hidrotermal [11], reacción sol-gel [12] y síntesis mecano-química [13]. Esta última técnica ha sido empleada en las últimas décadas para producir una variedad de fases tales como compuestos

\footnotetext{
*Correspondig author.e-mail: jquispem@unmsm.edu.pe
} 
intermetálicos, aleaciones amorfas, nanocristalinas, cuasicristalinas, y soluciones sólidas supersaturadas. En el presente trabajo se reporta un estudio comparativo entre una bioapatita, obtenida a partir de un tejido óseo humano, y compuestos de Hap obtenidos por precipitación química y síntesis mecano-química. Ambas muestras fueron comparadas con muestras comerciales de Hap existentes en el mercado. El presente trabajo esta organizado de la siguiente manera: en la sección I presentamos una breve introducción sobre Hap. En la sección II se indican los materiales y métodos de análisis empleados para el estudio de la Hap. En la sección III se reportan los resultados y una discusión de los mismos y finalmente en la sección IV se presentan las conclusiones obtenidas en el presente trabajo.

\section{Materiales y Métodos}

\subsection{Hidroxiapatita comercial}

Se analizó una muestra de hidroxiapatita marca Ostim $^{\text {TM }}$ (ver Tabla 1). Previamente esta muestra fue secada en una mufla a $60{ }^{\circ} \mathrm{C}$ durante 12 horas, pulverizada en un mortero y tamizada hasta un tamaño menor a $45 \mu \mathrm{m}$. Luego fue tratada térmicamente a $900^{\circ} \mathrm{C}$ durante 6 horas (Ver tabla 1).

\subsection{Preparación del tejido óseo.}

Se analizó la componente inorgánica de una muestra de hueso fémur humano de un paciente masculino de 50 años, quien fue sometido a un transplante de cabeza de fémur en un hospital local. Antes de los análisis la muestra fue desproteinizada por inmersión en $\mathrm{NaOCl}$ al 6\% durante 30 horas. Luego la muestra fue lavada con agua destilada y secada en una mufla a $60{ }^{\circ} \mathrm{C}$ durante 12 horas, para luego ser pulverizada. Posteriormente la muestra pulverizada fue tratada térmicamente a $900^{\circ} \mathrm{C}$ durante 6 horas (ver Tabla 1).

Tabla 1 Codificación de las muestras estudiadas.

\begin{tabular}{|c|c|}
\hline Identificación & Muestra \\
\hline MP01 & $\begin{array}{l}\mathrm{CaCO}_{3} \text { obtenida de la cáscara de huevo } \\
\text { de gallina }\end{array}$ \\
\hline MP02 & $\left(\mathrm{NH}_{4}\right) \mathrm{H}_{2} \mathrm{PO}_{4}$ Fisher P.A \\
\hline HAP01 & $\begin{array}{l}\text { Muestra preparada por reacción mecano } \\
\text { química }\end{array}$ \\
\hline HAP02 & $\begin{array}{l}\text { Muestra preparada por precipitación } \\
\text { química }\end{array}$ \\
\hline HAP03 & Hidroxiapatita comercial marca OSTIM \\
\hline HAP04 & $\begin{array}{l}\text { Componente inorgánica del tejido óseo } \\
\text { humano (bioapatita) }\end{array}$ \\
\hline
\end{tabular}

\subsection{Preparación de hidroxiapatita}

C1.- Síntesis mecano-química: Mediante esta técnica se prepararon muestras de Hap empleando un contenedor de acero endurecido y bolas de acero inoxidable en un equipo de molienda de alta energía SPEX8000-MIXER MILL. Las materias primas empleadas fueron $\mathrm{CaCO}_{3}$, la cual es extraída de la cáscara de huevo de gallina, sumergiendo 100 gr de cáscara de huevo en $75 \mathrm{ml}$ de hipoclorito de sodio $\mathrm{NaOCl}$ al 5\% luego fue lavada con agua bidestilada y posteriormente secada en un horno a $50^{\circ} \mathrm{C}$. Se utilizo 3 gr de $\mathrm{CaCO}_{3}$ y 2 gr de fosfato de amonio difásico $\left(\mathrm{NH}_{4}\right) \mathrm{H}_{2} \mathrm{PO}_{4}$ (Fisher P.A). a reacción mecanoquímica se realizó siguiendo la reacción en el equilibrio:

$$
\begin{gathered}
10 \mathrm{CaCO}_{3}+6\left(\mathrm{NH}_{4}\right) \mathrm{H}_{2} \mathrm{PO}_{4} \rightarrow \\
\mathrm{Ca}_{10}\left(\mathrm{PO}_{4}\right)_{6}(\mathrm{OH})_{2}+8 \mathrm{H}_{2} \mathrm{O}+10 \mathrm{CO}_{2}+6 \mathrm{NH}_{3}
\end{gathered}
$$

La reacción mecano-química se interrumpió después de 30 horas de molienda. La muestra así obtenida fue secada, pulverizada y tratada térmicamente a $900^{\circ} \mathrm{C}$ durante 6 horas antes de ser analizada (Ver tabla 1).

C2.- Precipitación química: Mediante este método se prepararon muestras de Hap empleando los reactivos $\mathrm{Ca}\left(\mathrm{NO}_{3}\right) \cdot 4 \mathrm{H}_{2} \mathrm{O}$ (Sigma Q.P) y $\left(\mathrm{NH}_{4}\right) \mathrm{H}_{2} \mathrm{PO}_{4}$ (Fisher P.A) en concentraciones $1 \mathrm{M}$ y $0.48 \mathrm{M}$, respectivamente. Antes de producir la reacción, se basificó los reactantes con amonio $\mathrm{NH}_{4}$ dejando precipitar $100 \mathrm{ml}$ de $\mathrm{Ca}\left(\mathrm{NO}_{3}\right) \cdot 4 \mathrm{H}_{2} \mathrm{O}$ sobre $10 \mathrm{ml}$ de $\left(\mathrm{NH}_{4}\right) \mathrm{H}_{2} \mathrm{PO}_{4}$. La síntesis se realizó siguiendo la reacción en el equilibrio:

$$
\begin{gathered}
10 \mathrm{Ca}\left(\mathrm{NO}_{3}\right) .4 \mathrm{H}_{2} \mathrm{O}+6\left(\mathrm{NH}_{4}\right) \mathrm{H}_{2} \mathrm{PO}_{4}+14 \mathrm{NH}_{4} \mathrm{OH} \rightarrow \\
\mathrm{Ca}_{10}\left(\mathrm{PO}_{4}\right)_{6}(\mathrm{OH})_{2}+20 \mathrm{NH}_{4} \mathrm{NO}_{3}+52 \mathrm{H}_{2} \mathrm{O}
\end{gathered}
$$

Luego de la reacción la muestra se dejó reposar a temperatura ambiente por 120 horas. La muestra así obtenida fue lavada y secada a $60^{\circ} \mathrm{C}$ durante 4 horas, pulverizada en un mortero y posteriormente tratada térmicamente a $900^{\circ} \mathrm{C}$ durante 6 horas (ver tabla 1).

La caracterización estructural de las muestras se realizó mediante difracción de rayos $\mathrm{X}$ (DRX) en un equipo D8 Focus-Bruker, empleando un tubo de $\mathrm{Cu}$, usando una radiación $\mathrm{K} \alpha_{1}$. El análisis por espectroscopia infrarroja (FTIR) se realizó en un espectrómetro NICOLET, Impact 410 usando $\mathrm{KBr}$ para la preparación de las muestras

\section{Resultados y Discusión}

El análisis por difracción de rayos $\mathrm{X}$ de los compuestos empleados como materia prima durante la síntesis de Hap permitió caracterizar las fases del 
$\mathrm{CaCO}_{3}$ y $\left(\mathrm{NH}_{4}\right) \mathrm{H}_{2} \mathrm{PO}_{4}$ identificadas por las fichas PDF-ICDD: \#89-1304 y PDF-ICDD: \#37-1479, respectivamente. Este análisis permitió identificar que el $\mathrm{CaCO}_{3}$ presenta impurezas de magnesio en su estructura.

En la Fig. 1, se muestra la comparación entre los difractogramas de la bioapatita (HAP04) respecto de la hidroxiapatita sintetizada por diferentes técnicas: precipitación química (HAP02) y síntesis mecano química (HAP01). Así también se presenta un difractograma de una muestra comercial de hidroxiapatita (HAP03).

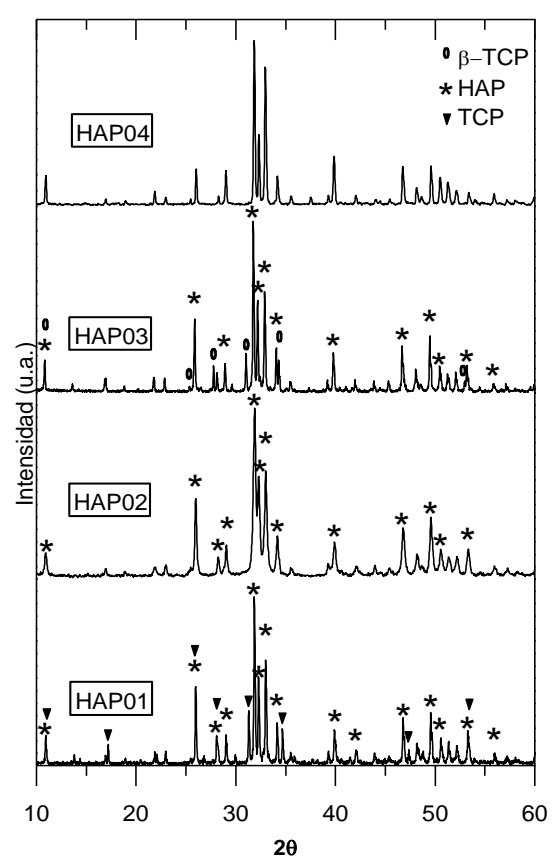

Fig. 1. Difractogramas de las apatitas obtenidas a partir de las muestras HAP01, HAP02, HAP03 y HAP04.

Los resultados obtenidos mostraron que la muestra HAP01 luego del tratamiento térmico se descompone en dos fases; una de ellas corresponde a una fase de Hap identificada con la ficha PDF-ICDD: \#89-6439 y la otra corresponde a una fase de fosfato de calcio (TCP) con inclusiones de hierro y magnesio identificada con la ficha PDF-ICDD: \#79-2186. El análisis de la muestra de hidroxiapatita comercial mostró la presencia Hap (PDF-ICDD:\# 84-1998) y $\beta$ TCP (PDF-ICDD:\# 70-2065) mientras que en la muestra HAP02 solamente se identificó una sola fase correspondiente a Hap (PDF-ICDD:\# 84-1998).

La Fig. 2, muestra la comparación entre los espectros FTIR de las muestras estudiadas. Todos los espectros presentan bandas de absorción alrededor de $875 \mathrm{~cm}^{-1}, 1420 \mathrm{~cm}^{-1}$ y $1480 \mathrm{~cm}^{-1}$, los cuales son atribuidos a los modos vibracionales del ión carbonato ( $\mathrm{CO}_{3}^{2-}$ ) sustituido en los sitios del fosfato [13-14]. Se observa además que la cantidad de carbonato presente en la muestra HAP01 y HAP04 es mayor respecto a las muestras HAP02 y HAP03. La presencia del carbonato en HAP04 es mucho mayor que en las otras muestras. Vale la pena mencionar que algunos investigadores reportan que la presencia de carbonato en pequeñas cantidades en apatitas biológicas es muy común y esta correlacionado con la actividad metabólica de la bioapatitas [14-15].

Las bandas anchas observadas alrededor de 1630 $\mathrm{cm}^{-1}$, y $3430 \mathrm{~cm}^{-1}$ indican la presencia de $\mathrm{H}_{2} \mathrm{O}$ absorbida por la muestra. Mientras que las bandas observadas a $634 \mathrm{~cm}^{-1}$ y $3570 \mathrm{~cm}^{-1}$ se producen debido al $\mathrm{OH}$ estructural. Las bandas de absorción debidos al $\mathrm{PO}_{4}{ }^{3-}$, se ubican alrededor de $570 \mathrm{~cm}^{-1}$, $605 \mathrm{~cm}^{-1}, 964 \mathrm{~cm}^{-1}, 1040 \mathrm{~cm}^{-1}$ y $1090 \mathrm{~cm}^{-1}$.

Los resultados anteriores indican que las muestras corresponden a apatitas deficientes en calcio; donde la muestra HAP01 corresponde a una apatita tipo $\mathrm{AB}$ [15-16] en donde se produce una sustitución parcial de iones $\mathrm{PO}_{4}{ }^{3-}$ por $\mathrm{CO}_{3}{ }^{2-}$.



Fig. 2. Espectros FTIR de las muestras HAP01, HAP02, HAP03 y HAP04.

\section{Conclusiones}

Los resultados obtenidos indican que todas las muestras poseen características estructurales similares. Notándose además que la muestra 
sintetizadas por reacción mecanoquímica posee características muy similares a la componente inorgánica del tejido óseo. Los resultados también muestran la posibilidad de sintetizar hidroxiapatita empleando como materia prima cáscara de huevo de gallina. En un futuro próximo se planea realizar un estudio más detallado con el objetivo de determinar la influencia de las impurezas sobre la estructura de la Hap durante la síntesis mecanoquímica.

\section{Agradecimientos}

Los autores agradecen al VRI-UNMSM por el apoyo financiero mediante el proyecto multidisciplinario $\mathrm{N}^{\mathrm{O}}$ PEM2006B19 y al VRADUNMSM por el apoyo financiero mediante el PIC.

\section{References}

[1]. R.Z. LeGeros:., Prog Crys. Growth Charact. 4 (1981) 1

[2]. W. Suchanek, M. Yoshimura., J. Mater. Res. Soc. 13 (1998) 94.

[3]. T. M. G. Chu, J. W. Halloran, S. J. Hollister, S. E. Feinberg. J. Mater. Sci.: Mater. Med. 12 (2001) 471

[4]. O. R. Costa, F.J. Veinsten., Rev. Asoc. Odontol. Argent. 82 (1994) 117.

[5]. G.G. Massry, J.B. Lodz., Ophthalmology 102(1) (1995) 161.
[6]. C. Klein, P. Patka and W. den Hollander, Biomaterials 10 (1989) 59.

[7]. K. de Groot, "Ceramic of Calcium Phosphates: Preparation and Properties, in Bioceramics of Calcium Phosphate", ed. K. de Groot (CRC Press, Boca Raton, FL., 1983) 100-114.

[8]. T. Ban, M. Akao, H. Aoki, and S. Iwai, (1977); Thermal analysis and calcified tissues Reports of medical and dental engineering, Tokyo Medical and dental University, Vol. 11 pp. 2731.

[9]. E. Rivera, R. Curiel, R. Rodiguez, Mat. Res. Innovat 7 (2003) 85

[10]. S. Puajindanetra, S.M. Best and W. Bonfield, Br. Ceram. Trans. J., 93 (1994) 96.

[11]. W. Suchanek, H. Suda, M. Kahihana, and M. Yoshimura, J. Mater. Res., 10 (1995) 521.

[12]. A. Deptula, W. Lada, T. olczak, A. Borello, C. Alvani and A. Dibartolomeo, J. Non-Cryst. Solids, 147 (1992), 537.

[13]. M. Toriyama, A. Ravaglioli, A. Krajewski, G. Celotti and A. Piancastelli, J. Eur. Ceram. Soc., 16 (1996) 429.

[14]. A. Stch, W. Jastrzebski, A. Brosek, J. Stoch, J. Szaraniec, B. Trybalska and G. Kmita., J. Mol. Struct. 555 (2000) 375.

[15]. J. S. Oleksiak, B. Mycek, A. Slosarczyk., Biomaterials. 21 (2000) 1215.

[16]. E. Landi, G. Celotti, G. Goehl, I.R. Dickson and A. Tampieri., J. Europ. Ceram. Soc. 23 (2003) 2931. 